\title{
El desarrollo histórico del concepto bibliografía
}

\author{
VALENTINO MORALES LÓPEZ \\ Doctorando en Bibliotecología y Estudios de la Información \\ FFyL-CUIB, becario de la Dirección General de \\ Estudios de Postgrado de la UNAM. \\ E-mail:dassos@hotmail.com
}

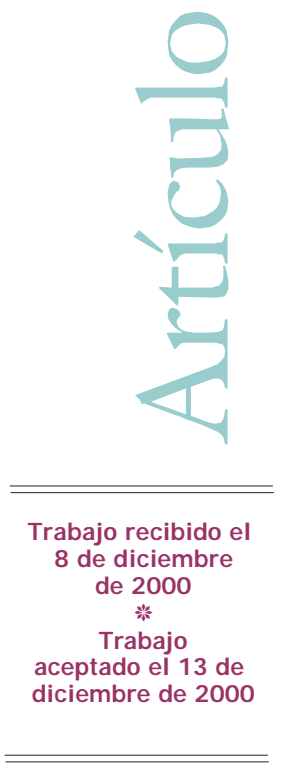

\section{RESUMEN}

Se presentan, analizan y discuten diversas connotaciones que ha tenido el término bibliografía lo largo de la historia. Entre éstas destacan las nociones de bibliografía como lista de obras; como una de las disciplinas encargadas de estudiar el libro; como rama de la bibliotecología o de otra disciplina documental; o como ciencia independiente que posee su propio aparato teórico y tiene su campo en la crítica de textos.

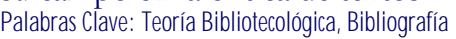

\section{THE HISTORICAL DEVELOPMENT OF THE CONCEPT BIBLIOGRAPHY VALENTINO MORALES-LÓPEZ}

\begin{abstract}
The diverse connotations of the term bibliography throughout history are presented, examined and discussed. Among these concepts the most notable are: the bibliography as a list of works; as a discipline dedicated to the study of books; as a branch of Library Science; or as a documentary discipline which possesses its own theoretical apparatus and which has a role to play in the critique of texts.

KeyWtros LibrariansipTheory, Bibliographies
\end{abstract}

\section{INTRODUCCIÓN}

$\mathbf{U}^{\text {nod }}$ no de los problemas teóricos que necesitan ser resueltos para permitir el ulterior desarrollo de la ciencia que estudia el campo de la información documental y objetos que aparecen en él, es el análisis de los conceptos que se manejan en este campo. El presente trabajo tiene como finalidad analizar uno de esos conceptos, que es el de bibliografía. Este artículo es parte de un programa de investigación de mayor envergadura que se está realizando dentro del Programa de Doctorado en Bibliotecología y Estudios de la Información de la UNAM, donde se analiza el desarrollo de otros términos relacionados con el estudio de la información registrada. 
En la actualidad son múltiples las nociones que se tienen en torno al término bibliografía. Entre ellas está aquella que la define como una mera lista de obras; y aquella que la considera como una de las disciplinas encargadas de estudiar al libro. Por su parte la comunidad bibliotecológica mexicana ubica a la bibliografía como una rama de la bibliotecología.

Ante esa variedad de percepciones se hace necesaria una revisión histórica del desarrollo del término bibliografía para tener una visión más clara de las diferentes nociones que se manejan. Tal revisión, además de ser exhaustiva, necesita ser selectiva, puesto que en muchas ocasiones una misma definición es retomada por varios autores. Así este trabajo se rige por los siguientes criterios:

1. Se presenta al primer autor que propuso una definición.

2. Se toma en cuenta un autor que alcanzó cierta importancia gracias a la innovación de sus ideas o a la influencia que tuvo entre los estudiosos de la bibliografía.

3. Cuando existe controversia se presentan los autores que son eje del debate.

El artículo consta de cuatro partes, en la primera de las cuales se presentan los orígenes del término bibliografía; en la segunda se dan a conocer cuáles fueron las primeras definiciones que se le adjudicaron a ese término; y en la tercera se muestra cómo fue consolidándose la noción de bibliografía como disciplina; en la cuarta y última parte se resalta la noción de bibliografía como uno de lo soportes de la investigación científica.

\section{LA BIBLIOGRAFÍA ENTENDIDA COMO UNA LISTA DE LIBROS}

Gracias a la consolidación de la imprenta de tipos móviles y a un vigoroso mercado de libros se inició una nueva etapa de la historia de la bibliografía. Era tiempo de reconocer la producción de bibliografías bajo una determinada nomenclatura.

La primera ocasión que apareció el término bibliografía para titular una obra que contenía una lista de libros fue en 1633, en el trabajo de Gabriel Naudé, Bibliographia Pditica(Naudé. El título de esta obra nos permite inferir que al término bibliografía se le asignaba el significado de lista descriptiva de libros o guía discursiva de los libros (Wdeege, p 267). Louis Jacob adopta esa idea y publica dos obras cuyo título contiene la palabra bibliografía y retiene la misma idea de Naudé. Tales obras son la Bibliographia pontifidade 1643 y la BibliographiaGallicaunivesalis publicada entre 1644 y 1645.

El sentido que Naudé le daba a la palabra bibliografía fue usado también en trabajos alemanes y uno de los primeros indicios data de 1705. Sin embargo continuaron siendo usados otros términos y a partir de 1703 biblidheafue el término que se usaba normalmente para las guías descriptivas de libros, no para las listas de libros. Pero bibliogaphiay sus equivalentes algunas veces fueron términos usados, entre 1641-1710 también para describir las listas de libros (Wdeege, $\mathrm{p}$ 267). 
En este primer momento del uso del término bibliografía (más no del concepto), el ambiente intelectual en Occidente ya había sufrido una fuerte transformación. La filosofía natural propuesta por Newton se había consolidado y el trabajo de investigación de la naturaleza ya gozaba de prestigio.

A pesar de que en este tiempo los principales eruditos estaban de acuerdo respecto de la existencia de principios universales que regían al universo se distinguían dos corrientes. Una de ellas continuaba la tradición humanista de los clásicos grecolatinos y por ende menospreciaba todo lo relacionado con el trabajo manual. La otra, aunque reconocía lo valioso de la tradición humanista y varios de esos autores se apoyaban en esa tradición para sostener sus ideas, exploraba el universo físico creyendo en un creador del universo. Para esta corriente lo único que restaba para comprender mejor el mundo era encontrar las leyes que regulaban ese universo físico.

En esta última corriente se distinguen dos escuelas:

1. La anglosajona, que tenía a Francis Bacon como principal ideólogo y quien se apoyaba en los estudios de la vieja escuela sajona de Occam. El pensamiento de Bacon influyó a la Royal Socieyy en consecuencia en el trabajo de Newton. La bibliografía de Francis Bacon proponía la denominación de literaryhistarypor primera vez en 1605, para mencionar una deseable rama de estudio que todavía no existía (Wdedge $\mathrm{p}$ 266-7). La Royal Society se encargó de elaborar importantes bibliografías temáticas, con el fin de apoyar la labor de sus miembros y de dar a conocer la producción bibliográfica de sus autores.

2. La otra corriente era la continental, con Rene Descartes a la cabeza. Éste reconocía la influencia de la vieja escuela parisiense de la Sorbona (Descartes, p. 82-86). El continuador de la obra de Descartes fue Leibniz, quien llegó a trabajar como bibliotecario y a quien algunos le atribuyen cierta influencia en el pensamiento bibliográfico y bibliotecario de su tiempo.

Pese a la existencia de importantes trabajos bibliográficos no se tiene noticia de que en éstos se haya propuesto alguna definición. La mayoría de los libros publicados sobre bibliografía eran en realidad listas de libros, algunas de las cuales incluían análisis del contenido de las obras. Por eso Georg Schneider afirma que en ese tiempo no hubo producción teórica.

\section{PRIMERAS DEFINICIONES PROPUESTAS PARA EL TÉRMINO BIBLIO- GRAFÍA}

La primera definición formal de la que se tiene noticia data de 1704 y sostiene que la bibliografía es un: conocimiento e interpretación de los antiguos manuscritos (Trévoux). Esta definición tuvo gran influencia entre los impulsores de la bibliografía. Posteriormente en 1774, el Diccionario de la Academia de la Lengua de Francia le adjudica al término el significado de: conocimiento de los manuscritos antiguos, definición con la que concuerda el Padre Terreros en 1786 (Terreros). Es interesante 
señalar que los creadores de la Enciclopedia, Diderot y D'Alembert, fueron fieles a la etimología del término pero sólo ofrecieron la definición de bibliógrafo y no la de bibliografía, sin aducir razones para tal decisión. Su definición de bibliógrafo era "toute personne versée dans la connaissance et le déchiffrement des manuscrits" (Malclés, 1950, t. 1, p. 1).

Ante todo es importante aclarar la necesidad de cocsiderar al concepto en su contexto histórico. Lo que lleva a preguntarse, ¿qué entendían esos primeros definidores por "conocimiento de los manuscritos"? Entendían que se trataba del estudio profundo de las características materiales e intelectuales de los manuscritos con el fin de conocer su valor. Esa manera de entender la bibliografía, se correspondería con la de la filología moderna. Pero, ¿dónde queda el libro impreso? Al parecer en ese momento no se valoraba demasiado al libro impreso, aunque se formaban numerosas listas, como el estudio de los manuscritos.

De cualquier manera el avance de la bibliografía era incontenible. En 1774, por primera ocasión, se usa el término bibliografía para referirse a la ciencia del libro. Esto lo propuso Michael Denis, director de la Biblioteca Imperial de Viena (Denis). Esa concepción la apoyó Francois Née de la Rochelle en 1782 (Rochelle), al que Simón Díaz considera como el primero que propuso el significado de ciencia del libro para la bibliografía. Pero como puede observarse en las fechas anotadas, la obra de Michel Denis es anterior por ocho años, por lo que podemos concluir que fue él primero en proponer esa definición.

Esta nueva definición de bibliografía amplió el espectro de estudio de esta disciplina, y la llevó a incluir varias áreas que en la actualidad no se le reconocen, pero ya que se consideraba que la bibliografía, al ser la ciencia del libro, estudiaba las bibliotecas, el mercado editorial, el amor a los libros, etcétera. Como puede verse se le atribuía un dominio hegemónico sobre el estudio de todos los asuntos relacionados con el libro.

Pero ¿por qué fue necesario que se propusiera una ciencia del libro? En ese momento comenzaba a tener importancia hablar de grandes ciencias que abarcaran el estudio de un fenómeno un objeto. Y para darle validez a esto surgieron las ciencias, aun éstas no contaban todavía con metodologías rigurosas. De hecho muchas de las modernas ciencias duras, estaban en ese momento dando a luz algunos de sus logros fundamentales.

Algo que influyó en esto fue el trabajo de los personajes de la Ilustración, quienes reunieron todo el conocimiento generado y lo plasmaron en la Enciclopedia. La importancia del trabajo de los creadores de la Enciclopedia para el campo bibliográfico radica en la mayor preponderancia que le dio al contenido intelectual de los libros por encima de su valor material. Asimismo Diderot y D'kambert forjaron parte del pensamiento que ejerció influencia en acontecimientos posteriores como la revolución francesa, el desplazamiento de la noción de un ser divino como eje del universo, y la visión del universo como una máquina. Otra idea importante fue la exaltación de la razón como principio de la naturaleza del ser humano. La razón, 
todopoderosa e infalible, ocuparía el lugar que antes tenía la providencia divina como motor del desarrollo del sujeto, y sería la que le permitiría pensar en un mundo ideal. Todo lo anterior sirvió de base para el surgimiento de las utopías sociales del siglo XIX.

Culminada la revolución francesa, el gobierno de la Convención apoyó el desarrollo de la bibliografía, entendida como ciencia del libro. En 1793 Urbain Domergue escribió un Rapapat, con el fin de concentrar los fondos de las bibliotecas nacionalizadas. Por su parte, Henri Gregoire presentó un informe a la Convención sobre el estado que guardaban las bibliotecas y sus fondos en la Francia postrevolucionaria. Ambos personajes, basados en las tesis de Francois Née de la Rochelle, llegaron a la conclusión de que al ser la ciencia del libro, la bibliografía era también la ciencia de las bibliotecas (Perales, p. 11-12).

Cuando Napoleón era emperador, se estableció que la bibliografía era la ciencia auxiliar de la historia, posición que se fortaleció con el paso del tiempo y que aún en la actualidad continúa gozando de seguidores (Escamilla, p. 114). Ese significado era comprensible porque ya que la mayoría de los bibliógrafos de ese tiempo tenían ocupaciones diversas y la bibliografía sólo era uno más de sus intereses intelectuales. Además no existe noticia de personas que se dedicaran únicamente a la bibliografía.

A pesar de que la posición francesa fue la más reconocida no era ciertamente la única, pues en esa misma época la postura prevaleciente entre los eruditos de lengua alemana era que la bibliografía trataba sobre la descripción del libro. Ese significado lo presenta Friedrich Adolf Ebert en su Allegemeines bibliographisches lexikon. La idea alemana de bibliografía es más cercana a la que se sostiene en la actualidad, lo que no quiere decir que necesariamente fuera la más acertada.

En estas primeras propuestas que intentan darle un concepto formal a bibliografía, se pueden apreciar tres posturas. La primera sostiene que la bibliografía es el conocimiento de los manuscritos; la segunda que es la ciencia del libro; y la tercera que es la descripción de los libros. Sin embargo casi todas las publicaciones relacionadas con la temática eran listas de libros y sus definiciones sólo se localizaban en diccionarios, enciclopedias o en las introducciones a los libros que contenían listados de libros.

El aporte inglés se dio especialmente en el terreno práctico, quizá en concordancia con su tradición filosófica. El trabajo más representativo fue el de Thomas Harwell Horne, Introductiontothestudy ffbibliogaphy, donde solo ocasionalmente se discute en torno de la bibliografía por esencialmente abordar la historia de la imprenta, de la escritura y de las artes del libro.

Sin embargo los países donde se dieron los aportes de mayor importancia para la bibliografía fueron Francia y Alemania. En Francia esto se debió a la antigua tradición de la bibliografía francesa. A pesar de la ruptura histórica producida por la revolución francesa, muchos de los personajes que influyeron para realizarla eran parte del movimiento de la Ilustración. En ese sentido el gobierno de la revolución en su afán de darle educación al pueblo procuró fortalecer áreas como la bibliografía. En el caso alemán es gracias al floreciente mercado de libros, no se debe olvidar que Gu- 
tenberg era de esa región, y a la formación de importantes bibliotecas. Además el interés por fundamentar teóricamente las áreas relacionadas con el libro recibió una atención especial.

\section{CONSOLIDACIÓN DEL TÉRMINO BIBLIOGRAFÍA}

En 1802 surgió una aparente voz disidente pero que concordaba con la visión de una ciencia del libro, Gabriel Peignot en su Didionnaireraisonnédebiblidogę aseveraba que la bibliografía era parte de la bibliología (Schneider, p. 5). Sostenía que al aumentar la producción de obras bibliográficas, la bibliografía alcanzaba un estado de madurez y que era necesario profundizar en su estudio. Por lo tanto se requería de una ciencia general que estudiara los principios elementales de la ciencia bibliográfica. Peignot nunca dejó de considerar a la bibliografía como una ciencia. De hecho en un trabajo posterior afirma que la bibliografía es unescienequi consistedanslaconnaissance

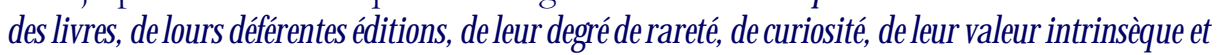
extrinsèque(cest-a dre), aux yeux dessavants edanslecommacedelalibraineetenfindurang quiis daivent ocuper danslesystemededassification adqptế (Peignot, p. xii).

Como se puede ver Peignot no rompe con la tradición bibliográfica francesa, su intención, más bién, es darle un mayor sustento científico a esta nueva disciplina. que a su juicio ya estaba consolidada. Al considerar que el aspecto práctico de la bibliografía era sólido, lo que buscaba con la introducción de la bibliología era conformar la parte teórica de la disciplina.

En los programas de la Ecole des Chartes, fundada mediante una ordenanza real fechada el 22 de febrero de 1821 (Martín, p. 529), bajo el término bibliografía se introdujeron contenidos relacionados con la historia de la imprenta y la clasificación, y en algunos momentos se incluía el estudio de las bibliotecas (Woledge, p. 267). Obviamente esta escuela recogía la tradición bibliográfica francesa.

El vigor de la bibliografía en Francia se vio reflejado en el hecho de que autores franceses aportaran los primeros manuales sobre bibliografía y que éstos contuvieran los primeros intentos por teorizar sobre el tema. Entre ellos resalta el libro de Francois Debure Bibliogaphieinstruative

Los franceses consideraron que ante el caos imperante entre las diversas colecciones de libros existentes en Francia surgía la necesidad de enfrentar esa problemática de manera creativa. Eso permitió que se le diera especial atención al desarrollo de la bibliografía y, como parte de ella, a la biblioteconomía. Lo que permitió el desarrollo de innovadoras teorías que se impartían en la naciente Ecole des Chartes (Martín, p. 531).

* Una ciencia que consiste en el conocimiento de los libros, de sus diferentes ediciones, de su grado de rareza, de curiosidad, de su valor intrínseco y extrínseco (esto es) a ojos de los conocedores, y en el comercio de la librería y, en fin, del rango que deben ocupar en el sistema de clasificación adoptado. Traducción del editor. 
Durante bastante tiempo la biblioteconomía se trató como una práctica subordinada a la bibliografía, lo que provocó que los pioneros de la biblioteconomía prestaran especial atención al desarrollo de técnicas de organización bibliográfica (Shera \& Egan, 1953, p. 15). Esa perspectiva se reprodujo en varios de los países europeos, entre ellos Italia y España.

Tiempo después Langlois concordaba con Peignot al considerar que la bibliografía era la parte de la ciencia de los libros que trataba de los repertorios y que facilitaba los medios para procurarse información sobre las fuentes (Langlois).

A diferencia de otras regiones, donde lo que se dejaba sentir era la influencia francesa, el significado más común de bibliografía en alemán fue el de listasdeliteratura Con la introducción de la biblidhksswissensdhatt (bibliotecología), la bibliografía se convirtió en una de las ramas de esa nueva ciencia. Esto provocó que las bibliografías preparadas por los bibliotecarios alemanes fueran diferentes a las preparadas por los estudiosos de la literatura y la historia (Woledge, p. 267).

Esa nueva perspectiva sobre la bibliografía trajo cierta confusión, pues a pesar de que se usaba el término bibliografía cada uno lo entendía de manera diferente. Los literatos e historiadores se enfocaban más al análisis del texto o la crítica literaria y a verificar la antigüedad y validez de las fuentes, en tanto que los bibliotecarios se concentraban en la conformación de repertorios bibliográficos. Confusiones de esta naturaleza han provocado largas y estériles discusiones, ya que cada uno presenta su concepción con base en el trabajo que realiza, pero no intenta verificar las diferentes partes que componen todo el concepto.

A pesar de que su labor en occidente no causó gran repercusión, se tuvo noticia de la labor de bibliógrafos de Europa oriental, así encontramos que entre 1850 y 1890, Grigorii Gennadi, un bibliográfo y bibliofilo ruso, sostenía decía que la bibliografía in thestrictsenseandnamowofthewadisbook description. Itstask istomakeknown, describe, andgivethehistary ofbooksandmanuscipts Itcanconsist finformationabattbooksof a partiaular peiod, arcauntry, arpeeple, ar ofinfomationabait a partiaular colletion of books (Choldin, p. 23). Como se puede observar esta definición de bibliografía coincide con la del alemán Adolf Ebert. Lo que trae en consecuencia que hasta ese momento sobre bibliografía se puede hablar, hasta este momento, de tres principales escuelas: la inglesa, con influencia sobre su antigua colonia, Estados Unidos; la francesa, con influencia en la zona mediterránea y en América Latina; y la alemana, que abarcaba el centro y este de Europa.

La idea de que la bibliografía era la ciencia del libro, prevaleció durante el siglo XIX. Muestra de ello es que Marcellin Berthelot define a la bibliografía como la ciencia de los libros desde el punto de vista de su descripción y de su clasificación (Escamilla, p.

* [...]en el estricto y estrecho sentido de la palabra es descripción de libros . Su tarea es hacer conocer, describir y dar la historia de libros y manuscritos. Puede también consistir de información sobre libros de un periodo particular, o un país ,o un pueblo, o de información acerca de una colección particular de libros. Traducción del editor. 
109). De acuerdo con Gloria Escamilla, esa definición fue copiada por la mayoría de los diccionarios. Lo interesante de esta definición es que en ella se conjugan los dos principales elementos que se le reconocen a la bibliografía: ciencia y descripción de los libros, a la que se añade su clasificación.

El elemento que llama la atención en la anterior definición de bibliografía es su mención explícita a la clasificación de los libros, aspecto que hasta el momento no había sido mencionado en las definiciones anteriormente presentadas en este trabajo. Esta cuestión es de suma importancia porque se trata de un elemento de la bibliografía que no está aislado sino en estrecha relación con los intentos por formar la clasificación de la ciencia.

En ese sentido, Jesse H. Shera considera que en su clasificación de la ciencia, Bacon, tiene como su antecedente más lejano a las taxonomías naturales formuladas por Aristóteles. Descartes también presenta un esbozo de la clasificación del saber humano en Prinipia philosqphiae, pero un trabajo que tiene especial interés es el de Leibniz, quien entre sus múltiples preocupaciones intelectuales cuenta con la formulación de una clasificación de la ciencia. Lo notable es que esos autores afirmaran que era posible hacer una clasificación universal del saber humano en general. Cuestión que en bibliografía adquiere especial interés porque permitiría una organización armónica de las listas de libros.

En consecuencia, al conformar los repertorios bibliográficos que contenían libros donde se encontraba el saber, los bibliógrafos consideraban que era posible crear una clasificación en la que cada libro podía tener su ubicación. Esta parte de la bibliografía cobró mayor auge durante el siglo XIX, ya que en los tiempos precedentes el ordenamiento de los textos en las bibliografías se hacía por orden alfabético. Por eso Schneider dice que la Biblicteabibliogaphicade Julius Petzhold, publicada en 1866, hace una gran aportación teórica a la bibliografía al incluir un listado preeliminar de esquemas de clasificación bibliográfica.

Este periodo es uno de los más ricos de la bibliografía. Durante la segunda mitad del siglo XIX son varios los libros que pretenden hacer aportaciones al desarrollo teórico de la bibliografía. Entre ellos se cuenta la introducción de Leopold Delisle al Catalogregenéral deslivesimpimésdelaBibliothequeNationalesen1 897; Giuseppe Ottino con su Bibliografía; W. T. Rogers A manual of Bibliography, de 1891; Friedrich Johann Kleemeier y su HanburhdarBibliogaphiede 1903. Todos con el común denominador de considerar a la bibliografía como la ciencia del libro que se ocupa de la descripción y clasificación.

Sin embargo la bibliografía se acercaba a otro momento crucial de su historia, que de alguna manera ya había sido anunciado por Peignot. Debido a que durante el siglo XIX habían surgido otras ciencias que tenían entre su objeto de estudio al libro, se subordinaba la vieja bibliografía a esas ciencias. Y quienes trabajaban en las nuevas ciencias consideraban que la elaboración de repertorios era una técnica, una herramienta o un método preponderante de su labor. 


\section{LA BIBLIOGRAFÍA VISTA COMO PARTE DE OTRAS CIENCIAS}

Hacia finales del siglo XIX y principios del XX, el factor dominante en el campo del saber es el tecnicismo. La visión de un mundo donde la máquina ocupa un lugar central en el mundo del ser humano es capital, de tal manera que se concibe al universo como una máquina que tiene un orden armónico. Aquí el positivismo fue en buena medida un factor determinante para generar esa idea, con su concepción de un universo con un orden perfecto.

A pesar de que incluso actualmente se tiene la idea de un saber global estructurado orgánicamente, un elemento comienza a tener mayor peso: la especialización y el apoyo al desarrollo de tecnología mediante amplios grupos de investigación. Esto acarrea consecuencias para la bibliografía, que comenzaron a ser notorias en ese tiempo y que se hicieron primero presentes en la producción bibliográfica y luego llegaron a afectar la noción misma de la bibliografía.

Pero con la especialización, la bibliografía enfrentó un grave problema que la hizo entrar en crisis, la llamada explosión documental. Fenómeno descrito como la excesiva producción de documentos y que provocó la imposibilidad de conformar bibliografías universales como la concebida por Gessner, y llevó a proponer las bibliografías nacionales y las especializadas.

Luego de este fenómeno la bibliografía volvió a restringirse al estudio de los repertorios o a la elaboración de listas de libros. Lo que se refleja en 1895, cuando La École des Chartes decidió separar lo relativo a los archivos y otras cuestiones, del ámbito de la nueva cátedra de Bibliografía. Charles Mortet, primer titular de la cátedra de bibliografía en la École, aseveraba que la misión de la bibliografía es: l'édudedes répetairesdanslesqudslesliviessontdénitsetdassésetauxquadsonditconstammentrecuirir sait paridantifierdeslives, saitpars enquéirdecequi aéépulbiésurunsuje* (Langlois, 1896). En ese mismo tiempo Langlois afirmaba que la bibliografía era aquella parte del estudio de los libros que trataba de los repertorios.

A finales del siglo XIX y principios del XX, al iniciar su proyecto de formar la bibliografía universal, Paul Otlet parte de la idea de que la bibliografía era una gran disciplina que hasta ese momento había sido circunscrita a desempeñar la modesta función de indicar fuentes bibliográficas (Otlet, 1990c, p. 14). Pero también le reconoce que tiene un pasado glorioso y ha logrado considerables progresos en el siglo XIX, con la compañía de la bibliología (Otlet, 1990a, p. 30-31).

En otro trabajo Otlet define a la bibliografía como aquella ciencia whosedgjet of studyisall thequestionscommm todifferentkindsofdbaments production, physical manufacture, distribution, inventary, statistics preservation, and useofbibliographicdouments that istosay,

* [...]el estudio de los repertorios en los que se describen los libros y a los que uno debe recurrir constantemente, ya sea para identificar los libros, o para preguntarse acerca de lo que ha sido publicado sobre un cierto tema. Traducción del editor. 


\section{eveythingwhidhdkalswithediting printing publishing book selling bibliographyandlibraryeeo namy' (Otlet, 1990b, p. 86).}

Otlet modifica gradualmente esa idea inicial hasta llegar a proponer que la bibliografía es una parte de la documentación y que la bibliología está encargada de los repertorios y de suministrar los medios para procurarse información sobre las fuentes (Otlet, 1907). Isabel de Torres considera que esa definición le sirve de base a Paul Otlet para desarrollar su idea sobre la documentación (Torres, p. 24).

La razón por la que Otlet llega a esas conclusiones es que no pensaba al libro como el único medio de comunicación entre los seres humanos. En su noción él consideraba que casi todos los objetos eran susceptibles de transmitir información. Por eso en su nueva propuesta incluía pinturas, vestigios históricos, documentos de archivos, revistas, etcétera. Además creía que era importante añadir también los nuevos medios de comunicación que se estaban generando, como la fotografía, los discos, el telégrafo, etcétera. En consecuencia notó la necesidad de contar con una nueva ciencia que no se restringiera sólo a los libros, y ésta era la bibliografía.

La propuesta de Otlet logró cierto consenso, en especial su idea de alcanzar una bibliografía universal. Sin embargo algunos estudiosos no aceptan que la bibliografía se subordine a la documentación, sobre todo aquellos que estudian el aspecto intelectual de los libros y se identifican con el área de las humanidades y las artes. Muestra de ello es la revisión publicada por Van Hoesen y Walter en 1928 (Shoemaker, p. 341).

En ese mismo tiempo Georg Schneider publica uno de los libros clásicos sobre la teoría e historia de la bibliografía del siglo XX, el Handauhderbibliographie En ese texto analizaba las fuentes más importantes que habrían de consultarse para estudiar la bibliografía, presentaba una historia de la bibliografía y revisaba el concepto; helo aquí: bibliographyas thestudy oflists ofliterature, thelists thendvesaregeneally temedbibliogaphies, andthir useistemmlbibliogaphing* (Schneider, p. 16). Como puede verse el concepto de Schneider no se aleja de las propuestas de la escuela alemana y envía la bibliografía hacia los estudios literarios, lo que se ve reflejado en el trabajo de Rudolf Blum.

Otro autor importante para la bibliografía de ese tiempo es el inglés Theodore Besterman, quien en su ensayo de 1936, Thebeginingof systematicbibliogaphy, diserta sobre la historia de la bibliografía y afirma que: Nousdefinionsdoncunebibiographiecommeune “listedelivesétabliesuivant un pinịpedretear constant." "*** (Bestermann, p. 11).

* [...]cuyo objeto de estudio son todas las cuestiones comunes a diferentes tipos de documentos: producción, manufactura física, distribución, inventario, estadística, preservación, y uso de documentos bibliográficos; es decir, todo aquello que tiene que ver con edición, impresión, publicación, venta de libros, bibliografía y economía bibliotecaria. Traducción del editor.

** [...]bibliografía como el estudio de listas de literatura; las propias listas son generalmente llamadas bibliografías, y su uso es denominado como hacer bibliografías. Traducción del editor.

*** Definimos, pues, a un bibliografía como una "lista de libros que se establece siguiendo un principio director constante." Traducción del editor. 
Preocupado por la ambigüedad del término bibliografía, evidente en los dos últimos conceptos expuestos, sir Walter Greg propone que debe ser definida como: the surdyofbodksasmaterial djets's (Greg). La razón de esa propuesta era para Greg la bibliografía, como estudio de los libros, era esencialmente la transmisión de los documentos literarios (Stdkes, p 16).

Para Martin Scheele la bibliografía es una disciplina que puede estar subordinada al campo de la bibliología, para lo cual cita a Totok, quien define a la bibliografía, como sigue: lists of writings established from cetain paints of view apart from thequestion of their availabilityin a library (therein distinguishedframa catalog; sudy ofliteraburereferening (structure, purpose, knoweelged thedifferentfoms, historical devdqpment); surdyofthepracticeof compilingsuchaids (themthoddogy of their construction)** (Scheele, p. 15-151).

Para la segunda mitad del siglo XX una de las propuestas que encontró mayor eco fue la de la francesa Louise Malcles, quien en un primer trabajo define a la bibliografía de la siguiente manera: La bibliographieetla connaissancedetauslestextespubliés au miltigraphiés Ellesefondesurlareherchel'identification, ladescription \&ledassement decesdo amentsenvuedorganiserdesservicsaudecontnuiredesintrumentsdestinésafailiterletravail in telletud ${ }^{* * *}$ (Malcles, 1950, t. 1, p. 3). En un trabajo posterior José Simón sostiene que: la bibliografía es una parte de la ciencia del libro o bibliología que se propone buscar, identificar, describir y clasificar los documentos impresos, a fin de construir repertorios apropiados para facilitar el trabajo intelectual (Simón, p. 18).

Simón considera a la bibliografía no como una ciencia autónoma sino como una rama de la bibliología; es notable la influencia de Peignot en esta noción de bibliografía. De hecho la misma Malcles en su libro Labibliografía, afirma que desde 1914 se inició la época técnica de la bibliografía. Y denomina a las otras épocas de la siguiente manera: siglo XVI, la época erudita; siglo XVII, la época histórica; siglo XVIII-1789, la época histórica y científica; 1790-1810, la época literaria y bibliofílica; 1810-1914, la época artesanal; y desde 1914, la época técnica (Malcles, 1960).

Examinando las propuestas de definición para bibliografía de Shneider, Bestermann y Malcles, que son los mas influyentes de la primera mitad del siglo XX, la conclusión es que en esa etapa los bibliógrafos habían dejado de considerar que la bibliografía fuera una ciencia. La posición que se le asignaba era la de ser una rama de

* $\quad[\ldots]$ el estudio de libros como objetos materiales. Traducción del editor.

** [...]listas de escritos establecidas con base en ciertos puntos de vista, además del asunto de su disponibilidad en una biblioteca (y por tanto diferenciadas de un catálogo); estudio referenciador de literatura (estructura, propósito, conocimiento de las formas diferentes, desarrollo histórico); estudio de la práctica de compilar tales ayudas (la metodología de su construcción) Traducción del editor.

*** La bibliografía es el conocimiento de todos los textos publicados o mulicopiados. Se funda en la investigación, la identificación, la descripción y la clasificación de estos documentos con miras a organizar servicios o a construir instrumentos destinados a facilitar el trabajo intelectual. Traducción del editor. 
otra ciencia mas general, o una actividad que apoyaba la labor de la ciencia (se le llegó a denominar ciencia auxiliar), o un método o herramienta del trabajo intelectual.

En mi opinión la mejor definición es la propuesta por Malcles, ya que no habla de manera general como Bestermann o Schneider, quienes se contentan con enunciar que la bibliografía es el estudio bajo un principio constante de listas, ya sea de la literatura o de libros, Malcles por un lado ubica a la bibliografía en las áreas del saber al decir que ésta es parte de la bibliología. Asimismo enuncia de manera general lo que es la bibliografía: el conocimiento de todos los textos editados o reproducidos mecánicamente. Además hace una descripción puntual de la labor de la bibliografía al señalar que se propone buscar, identificar, describir y clasificar los documentos impresos, a fin de construir repertorios apropiados para facilitar el trabajo intelectual (Simón, p. 18).

El mayor cuestionamiento para aceptar la definición de Malcles es que ubica a la bibliografía en el campo de la bibliología, ya que ésta es una ciencia que no es aceptada en países como Alemania y Estados Unidos. Sin embargo partiendo del contexto donde en que formula su propuesta Malcles, no existe tal problema, puesto que, como se vera en otra parte, en Francia la bibliología sí es aceptada como ciencia .

Además esa definición es importante para la comprensión posterior de la bibliografía, ya no considerada como ciencia sino como el conocimiento de todos los libros o de una rama del saber que se propone conformar listas de libros para apoyar la investigación; sobre todo porque era necesario dar respuesta a la aparición masiva de nuevos soportes de información que tenían igual validez que el libro y eran de interés para amplios sectores intelectuales. De hecho para quienes se encontraban involucrados en la investigación de punta, el libro dejó de ser el principal proveedor de información y fue sustituido, por las revistas en primer lugar y posteriormente por otros medios como la microficha o la fotografía.

\section{CONCLUSIONES}

La ambigüedad del término bibliografía es un problema al que pocas veces se le ha podido dar solución a través de la historia (Shoemaker, p. 340). Incluso hay autores, como Paul S. Dunkin, que aseveran que cualquier definición de bibliografía es una oración de experiencias personales o que se reduce al campo de la fe. El ejemplo que se cita es que en 1950 Percy Freer hizo una investigación que arrojó como resultado una lista de 50 definiciones, desde 1678 hasta 1900, y uno de sus resultados fue que pocas de esas definiciones concuerdan entre sí e incluso tienen serias diferencias (Harmon, p. 2).

La veracidad de los anteriores juicios es cercana a la realidad, sin embargo es necesario tener cuidado al aceptarlos porque, a pesar de la ambigüedad y la gran cantidad de definiciones que prevalecen en la bibliografía, se sabe bien que cada uno de 
esos significados se aplican de acuerdo con un contexto determinado. Por ejemplo si se está en un curso sobre métodos de investigación, cuando se menciona el término bibliografía el referente no será la ciencia del libro, sino aquella técnica que le permite al investigador poder profundizar en la producción intelectual de una línea de investigación determinada (Torre Villar).

Es posible considerar que por el momento existen tres posturas en torno a cuál es el carácter de la bibliografía. Una es aquella en que la bibliografía se subordina a otra disciplina, sea la bibliotecología, la documentación o la ciencia de la información. En este caso hay quienes van más allá y no la reconocen como entidad científica y sólo le adjudican el papel de instrumento, técnica o arte. Esta idea se generalizó porque en la investigación la bibliografía se presenta como una técnica o método que forma parte del método científico y que cobró fuerza a principios del siglo XX entre quienes realizan el trabajo bibliográfico como medio, mas no como fin.

La segunda perspectiva defiende el aspecto científico de la bibliografía y aunque acepta las relaciones que tiene con otras disciplinas, considera que se trata de una ciencia independiente que se regula con base es sus propias leyes. Aquí la bibliografía se da sobre todo en el campo de la crítica de textos ya sea desde un punto de vista histórico o literario, y se reconocen sus orígenes en el trabajo de Calímaco y más que nada en la bibliografía erudita. Sin embargo a pesar de que en esta postura se acepta el carácter independiente de la bibliografía, se la subordina a la historia, a la literatura y en algunos casos a la filosofía.

La tercera postura sostiene que la bibliografía es una rama de otra disciplina más general. Esta postura tuvo su origen en el trabajo de Peignot y con el paso del tiempo cobró fuerza entre quienes trabajan la bibliografía como fin y como medio. Entre las ciencias a las que se subordina la bibliografía están la bibliología, la bibliotecología, la documentación y la ciencia de la información.

Las definiciones que se dan en torno a la bibliografía son abundantes, muchas de ellas demasiado generales como para volver a ser mencionadas, o se trata de meras repeticiones de otras definiciones que sólo han sido puestas en otras palabras. Esta investigación considera que la mejor definición para la bibliografía es la de Malcles, siempre y cuando se omita que la noción de que la bibliografía pertenece al campo de la bibliología. Se acepta esta definición porque refleja la situación actual que vive la bibliografía: que se trata de un saber que sirve para conformar repertorios bibliográficos, y que hay un profundo conocimiento de los libros que se enlistan. Además coincide con el desarrollo histórico de la bibliografía que, con el paso del tiempo, dejó de ser una ciencia independiente y pasó a ser una rama de otras denáas como la bibliología, la bibliotecología, la documentación o la ciencia de la información.

Por lo tanto la bibliografía se debe definir como el conocimiento sobre los textos publicados. Y añadir que se ocupa de la búsqueda, la identificación, la descripción y la clasificación de esos documentos, con la intención de organizarlos para ofrecer servicios o para construir los instrumentos destinados a facilitar el trabajo intelectual. 


\section{BIBLIOGRAFÍA Y REFERENCIAS}

Besterman, Theodore. Lesdabutsdelabibliogaphiem thodique $-3^{\mathrm{a}}$ ed. - Paris : La Palme, 1950.

Blum, Rudolf. Bibliogaphia: aninquin intoitsdefinitionanddesigna tions - Chicago : University Press, 1980.

---. Kallimadhos: thealexandianlibraryandthearigins ofbibliography / tr. By Hans H. Wellisch. - Wisconsin : The university of Wisconsin Press, 1991.

Boulard, M. S. Traité Aémentaire debibliogaphie - Paris : Chez Boulard, 1845.

Chartier, Roger. El addendeloslibros Letores, autores, biblidteasen EuropaentrelossigosXIV yXVIII. - Barcelona: Gedisa, 1994.

Choldin, Marianna Tax. "Grigorii Gennadi and russian bibliography: a reexamination". En: Libri v. 25, n. 1, (1975)..

Denis, Michel. Gündissder bibliographie - Viena, 1774.

Descartes, Rene. "The meditations". p. 77-169. En: Rene Descartes. Them thod, meditations, and sdetions from thepriniples of Descartes - 10 ed. - Edinburgh : William Blackwood and Sons, 1890.

Downs, Robert B. "Foreword". En: Louis N. Ridenour; Ralph Shaw and Albert G. Hill. Bibliogaphyinan ageof siene - Illinois : University of Illinois Press, 1952.

Escamilla González, Gloria. "La bibliografía y el método bibliográfico". En: Bdetíndd InstitutodeInnestigaionesBibliogáficas n. 4, (jul.-dic., 1970).

Garza Ramos, Georgina Madrid. "Panorama de los cambios estructurales de la bibliografía contemporánea”. En: Anuariode Biblictedogáa Época IV, n. 3, (1982).

Greg, Walter. "Bibliography- a retrospect". En: TheBibliographical Socity, 1892-1942. Studiesinnerospet. London : Bibliographical Society, 1945.

Hamel, Christopher de. "Medieval library catalogues". En: Pio neessin bibliography: papes presenteelat a seminar inbodk tradehis tay, 27-28 noverber 1987 / ed. By Robin Myers and Michael Harris. - Hampshire : St. Paul's Bibliographies, 1988.

Harmon, Robert B.. Elementsoflibliogaphy: asimplifiedapproach New Jersey : The Scarecrow Press, 1981.

Langlois. Manud debibliographiehistarique - Austria : Akademische Druck, 1968. 
Malclés, L. N. Lessarcesdutravail bibliographique/ preface de Julien Cain. - Geneve : Libraire E. Droz, 1950.

Malcles, Louise. La bibligrafía - Buenos Aires : EUDEBA, c1960.

Martin, H.-J. "Les chartistes et les bibliotheques". En: Bullein desBiblictheques deFrance año 17, n. 12.

Mikhailov y Chernyi. Anintroductarycaurseaninfomatices doumentation - The Hague : FID, 1971.

Naudé, Gabriel. Bibliographia pditica: . - Paris : Guillaume Pele, 1642.

a. Otlet, Paul. "Creation of a universal bibliographic repertory: a preliminary note”. En: Seletel ssays of Paul Otle/ tr. and ed. with and intr. Boyd Rayward. - Amsterdam : Elseiver, 1990.

b. Otlet, Paul. "The science of bibliography and documentation" En: Sdetelessays ofPaul Otle/ tr. and ed. with and intr. Boyd Rayward. - Amsterdam : Elseiver, 1990.

c. Otlet, Paul. "Something about bibliography". En: SAeteles says ofPaul Otle / tr. and ed. with and intr. Boyd Rayward. Amsterdam : Elseiver, 1990.

Peignot, Gabriel. Répatairebibliographiqueunivesd - Paris : Chez Antoine-Augustin Renouard, 1812.

Perales de Mercado, Alicia. "La bibliografía: producto de sus autores y su tiempo". En: AnuariodeBiblidtedoǵa, Archivdoǵae Infomática época 3, año V, (1976).

Née de la Rochelle, Francois. Discaurssurlasciencebibliographique esurles desoirdubibligraphe Paris : Gogue et Nee de la Rochelle, 1782.

Scheele, Martin. Pundh-ard mthoos in research and dbamentation: withspecial referencetobidogy/tr. J. E. Homstrom. - New York : Interscience Publisher, 1961.

Schneider, Georg. Theary and histary of bibliography / tr. Ralph Shaw. - New York : Columbia University Press, 1934.

Shaw, Ralph R. "Machines and the bibliographical problems of the twentieth century". En: Louis N. Ridenour; Ralph Shaw and Albert G. Hill. Bibligraphyin an age of scieme - Illinois : University of Illinois Press, 1952.

Shera, Jesse H. Thefandations of elucation for libranianship - New York : Wiley \& Sons, c1972.

- - -. Introduction tolibrary sience basic dements of library saence Colorado : Libraries Unlimited, 1976. 
Shera, Jesse H. \& Margaret Egan. "Foundations of a theory of bibliography”. En: LibraryQuartely. v. 22, (april, 1952).

- - -. "A review of the present state of librarianship and documentation". En: S. C. Bradford. Doumentation - $2^{\text {nd }}$ ed. London : Crosby, 1953.

Shoemaker, Richard H.. "Bibliography (general)". En: Library Trends v. 15, n. 3, (jan., 1967).

Símon Díaz, José. Labibligrafía: coneptosyaplicadiones - Barcelona: Planeta, 1971.

Stokes, Roy. Thefunction of bibliography. - London : A Grafton Book, 1969.

Taffarelli, Jean-Louis. "Pour une redéfinition de la bibliographie”. En: BulleinBibl. France t. 25, n. 7, (1980).

Terreros y Pando, Esteban. Dicionario castellano con las voces de deniasyartesysuscomespondientesenlastreslenguas, francesa, latina eitaliana - Madrid, 1786.

Torre Ramírez, Isabel de la. Quéeslabibliografia: introducción para estudiantesdebiblideconomáaydoumentadón - Granada : Universidad de Granada, 1996.

Torre Villar, Ernesto de la. MÁdbyténicasdeinvestigacón - México : UNAM, 1970.

Woledge, G. "Bibliography and documentation: words and ideas". En: Jaumal of Doumentation v. 39, n. 4, (dec. 1983). 